\title{
Westerlund 1: monolithic formation of a starburst cluster
}

\author{
Ignacio Negueruela ${ }^{1}$, J. Simon Clark ${ }^{2}$, \\ Ben W. Ritchie ${ }^{2}$ and Simon P. Goodwin ${ }^{3}$ \\ ${ }^{1}$ Departamento de Física, Ingeniería de Sistemas y Teoría de la Señal \\ Escuela Politécnica Superior, Universidad de Alicante \\ Carretera de San Vicente del Raspeig s/n, E03690, San Vicente del Raspeig, Alicante, Spain \\ email: ignacio.negueruela@ua.es \\ ${ }^{2}$ Department of Physics and Astronomy, The Open University, Walton Hall \\ Milton Keynes MK7 6AA, United Kingdom \\ ${ }^{3}$ Department of Physics and Astronomy, University of Sheffield, Sheffield, S3 7RH, UK
}

\begin{abstract}
Westerlund 1 is in all likelihood the most massive young cluster in the Milky Way, with a mass on the order of $10^{5} M_{\odot}$. To determine its bulk properties we have made multi-epoch radial velocity measurements for a substantial fraction of its OB stars and evolved supergiants and obtained multi-object spectroscopy of candidate cluster members in its locale. The results of these two studies show that Westerlund 1 is apparently subvirial and appears completely isolated, with hardly any massive star in its vicinity that could be associated with it in terms of distance modulus or radial velocity. The cluster halo does not extend much further than five parsec away from the centre. All these properties are very unusual among starburst clusters in the Local Universe, which tend to form in the context of large star-forming regions.
\end{abstract}

Keywords. open cluster and associations: Westerlund 1, stars: formation

Star-forming regions in the Galaxy and beyond demonstrate hierarchical structure in both time and space, whereby star clusters form in larger complexes within their natal giant molecular clouds. A prime example is the galactic G305 complex, which consists of a number of proto-clusters embedded in the periphery of a wind-blown bubble driven by the young massive clusters Danks 1 and 2 (Clark et al. 2004; Davies et al. 2012). A further, diffuse population of isolated massive stars is distributed across the complex, with their properties implying that star formation has occurred for several Ma and is still proceeding apace (delineated by multiple deeply-embedded proto-stars/clusters, maser emission and compact $\mathrm{H}$ II regions).

However, our data on Westerlund 1 (Wd1) appear to tell a different story. Observations of evolved OB stars imply a highly uniform cohort, evolving continuously from O9 III to B2.5 Ia (e.g. Negueruela et al. 2010), consistent with a simple homogeneous, co-eval 5Ma stellar population, also accommodating the simultaneous presence of red supergiants and Wolf-Rayets (Clark et al. 2005). The apparent instantaneous star-forming event suggested by the massive stars within $\mathrm{Wd} 1$ is also echoed by observations of the main sequence turn-on, implying an age-spread of < 0.4 Ma (Kudryavtseva et al. 2012).

So how did Wd1 form? To answer this question we adopted a three-pronged approach:

- A large multi-epoch and -object spectroscopic survey undertaken to identify binary and pulsating stars (see Ritchie et al. 2009 and Fig. 1) also allowed us to distinguish those cluster members with constant radial velocity and thus determine the mean radial velocity dispersion of the cluster. This reveals a velocity dispersion of $<4.6 \mathrm{~km} \mathrm{~s}^{-1}$ (Clark et al. 2014 and in prep.), implying that Wd1 is sub-virial despite the fact that, given its 


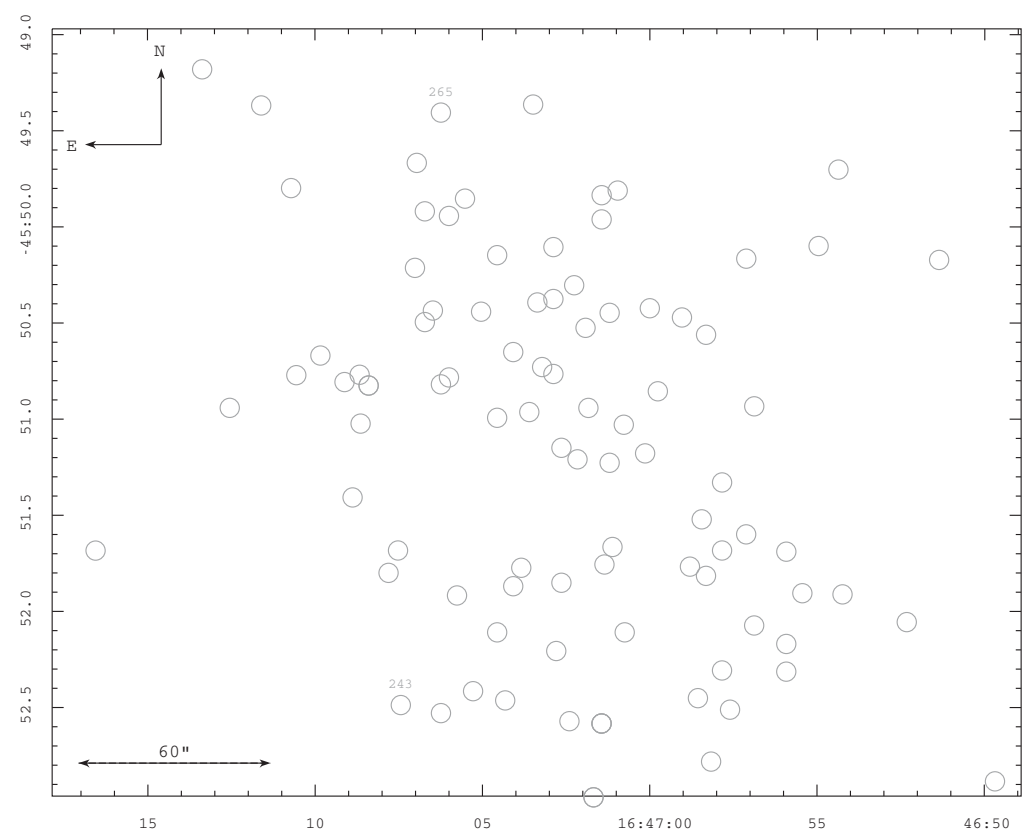

Figure 1. Finding chart for targets observed with FLAMES on the VLT for our radial velocity survey in the central regions of the cluster.

age and radial extent, one would anticipate that it should be dynamically relaxed and in virial equilibrium.

- Optical and near- to mid-IR wide-field imaging (e.g. Spitzer GLIMPSE/MIPSGAL) reveals no indication of satellite clusters, remnant giant molecular cloud nor ongoing star formation on spatial scales comparable to other star forming complexes such as G305.

- AAOmega multi-object spectroscopic observations were made of photometrically identified candidate early-type stars in the vicinity of $\mathrm{Wd} 1$; these revealed no evidence for a spatially extended "halo population".

Given these observations it appears that Wd1 formed in a single, compact and spatially isolated "starburst". Such a conclusion raises a number of important questions, such as (i) how can one accumulate so much mass in such a compact region?, (ii) why do we currently find Wd1 in a sub-virial state?, and (iii) why does it appear to have formed in a different manner compared to other massive stellar aggregates - does it simply represent an extreme example of a process which yields a continuum of cluster complexes/associations in terms of densities, spatial extents and age-spreads or is a different physical process responsible for its formation? In any event the sub-virial nature of Wd1 implies long-term survivability and so that it may represent the birth of a proto-globular cluster in the local Universe.

\section{References}

Clark, J. S. \& Porter, J. M. 2014, A\& A, 565, A90

Clark, J. S., Negueruela, I., Crowther, P. A., \& Goodwin, S. P. 2005, A\&̈A, 434, 949

Clark, J. S., Ritchie, B. W., Najarro, F., Langer, N., \& Negueruela, I. 2014, A\&A, 565, A90

Davies, B., Clark, J. S., Trombley, C., et al. 2012, MNRAS, 419, 1871

Kudryavtseva, N., Brandner, W., Gennaro, M., et al. 2012, ApJL, 750, L44

Negueruela, I., Clark, J. S., \& Ritchie, B. W. 2010, A\& 4 , 516, A78

Ritchie, B. W., Clark, J. S., Negueruela, I., \& Crowther, P. A. 2009, A\& A, 507, 1585 